Article

\title{
Biodiversity and Spatial-Temporal Dynamics of Margalefidinium Species in Jiaozhou Bay, China
}

\author{
Shuya Liu ${ }^{1,2,3,+}$, Mengjia Zhang ${ }^{1,2,3,4,+}$, Yongfang Zhao ${ }^{4,5}$ and Nansheng Chen $1,2,3,6, *$ (D) \\ 1 CAS Key Laboratory of Marine Ecology and Environmental Sciences, Institute of Oceanology, \\ Chinese Academy of Sciences, Qingdao 266071, China; liushuya@qdio.ac.cn (S.L.); \\ zhangmengiia@qdio.ac.cn (M.Z.) \\ 2 Functional Laboratory of Marine Ecology and Environmental Science, \\ Qingdao National Laboratory for Marine Science and Technology, Qingdao 266237, China \\ 3 Center for Ocean Mega-Science, Chinese Academy of Sciences, Qingdao 266071, China \\ 4 College of Marine Science, University of Chinese Academy of Sciences, Beijing 100039, China; \\ yfzhao@qdio.ac.cn \\ 5 Jiaozhou Bay National Marine Ecosystem Research Station, Institute of Oceanology, \\ Chinese Academy of Sciences, Qingdao 266071, China \\ 6 Department of Molecular Biology and Biochemistry, Simon Fraser University, Burnaby, BC V5A 1S6, Canada \\ * Correspondence: chenn@qdio.ac.cn \\ + These authors contributed equally to this work.
}

Citation: Liu, S.; Zhang, M.; Zhao, Y.; Chen, N. Biodiversity and Spatial-Temporal Dynamics of Margalefidinium Species in Jiaozhou Bay, China. Int. J. Environ. Res. Public Health 2021, 18, 11637. https:// doi.org/10.3390/ijerph182111637

Academic Editor: Pedro M. Costa

Received: 25 September 2021

Accepted: 4 November 2021

Published: 6 November 202

Publisher's Note: MDPI stays neutral with regard to jurisdictional claims in published maps and institutional affiliations.

Copyright: (C) 2021 by the authors Licensee MDPI, Basel, Switzerland. This article is an open access article distributed under the terms and conditions of the Creative Commons Attribution (CC BY) license (https:/ / creativecommons.org/licenses/by/ $4.0 /)$

\begin{abstract}
Many Margalefidinium species are cosmopolitan harmful algal bloom (HAB) species that have caused huge economic and ecological damage. Despite extensive research on Margalefidinium species, the biodiversity and spatial-temporal dynamics of these species remain obscure. Jiaozhou Bay is an ideal area for HAB research, being one of the earliest marine survey areas in China. In this study, we carried out the first metabarcoding study on the temporal and spatial dynamics of Margalefidinium species using the $18 \mathrm{~S}$ rDNA V4 region as the molecular marker and samples collected monthly at 12 sampling sites in Jiaozhou Bay in 2019. Two harmful Margalefidinium species (M. polykrikoides and M. fulvescens) were identified with potentially high genetic diversity (although we cannot rule out the possibility of intra-genome sequence variations). Both M. polykrikoides and M. fulvescens demonstrated strong temporal preference with a sharp peak of abundance in early autumn (September), but without showing strong location preference in Jiaozhou Bay. Our results revealed that temperature might be the main driver for their temporal dynamics. Knowledge of biodiversity and spatial-temporal dynamics of the Margalefidinium species may shed light on the understanding of mechanisms underlying strongly biased occurrences of Margalefidinium blooms recorded globally.
\end{abstract}

Keywords: Margalefidinium; metabarcoding analysis; Jiaozhou Bay; harmful algal bloom; spatial-temporal dynamics

\section{Introduction}

The genus Margalefidinium, which contains five species, namely, M. polykrikoides, $M$. fulvescens, $M$. catenatum, $M$. citron, and $M$. flavum, was recently transferred from the genus Cochlodinium on the basis of morphological and phylogenetic analyses [1]. It belongs to the family Gymnodiniaceae, order Gymnodiniales, class Dinophyceae, and phylum Miozoa [2]. Among these five described Marglefidinium species, two species, M. polykrikoides and $M$. fulvescens, have drawn particularly wide attention and have received extensive research primarily for their ability to form harmful algal blooms (HABs) [1,3]. Each of the five species has an eyespot in the episome that may be important for photoreception. Both of them are large $(>40 \mu \mathrm{m})$ and unarmored, commonly form chains, and have shallow sulcus without harboring longitudinal flagellum [4,5]. They differ in chloroplast shape, eyespot, and the position of sulcus, which are discernible under light microscopy, and in morphology 
of apical groove, which is discernible only under scanning electron microscope (SEM) [6,7]. However, these two HAB species cannot be easily distinguished morphologically under light microscope (LM) since they share similar morphological features with few differences.

Both M. polykrikoides and M. fulvescens are widespread, with populations being documented in tropical and warm-temperate waters around the world, and blooms being expanding in scope, respectively [8]. The first blooms of M. polykrikoides were detected in Southeast Asia and the east coast of North America before 1990 [6,9,10]. Since then, M. polykrikoides blooms have expanded to the East China Sea, the Philippines, Malaysia, Australia, the west coast of North America, Costa Rica, Latin America, Europe, and the Arabian Gulf region [7,8,11]. M. fulvescens has been reported to form massive blooms on the coast of Vancouver Island, Canada, and along the California coast, USA [12,13]. HABs caused by M. polykrikoides and M. fulvescens were not only strongly ichthyotoxic, but also capable of killing many other marine organisms [7]. In particular, M. polykrikoides had caused destructive blooms on a global scale, which were responsible for mass mortalities of wild and farmed fish, with catastrophic impact on local fishery operations, tourism, and economies [7,11]. Similarly, $M$. fulvescens blooms have caused mass mortalities of farmed salmon and mussels $[12,13]$. Although the mechanisms of toxicity of M. polykrikoides to fish have yet to been fully validated, they may involve many substances, such as reactive oxygen species (ROSs), hemolytic and neurotoxic-like substances, and extracellular mucoid polysaccharide substances $[8,14,15]$. Further studies showed that sonicated cultures and cell-free culture media of $M$. polykrikoides exhibited significantly reduced toxicity, suggesting that toxins were unstable under extreme physical conditions [16]. M. fulvescens is difficult to culture, and there are relatively few studies on its harmful effects; some studies suggested that $M$. fulvescens possesses ichthyotoxic properties similar to those of M. polykrikoides [5,17].

Characterization of Margalefidinium species has been carried out largely using microscopical methods. The life cycle of M. polykrikoides was detected in monoclonal isolation and morphological observation, indicating two different stages (an armored and an unarmored vegetative stage) [18]. Resting cysts have been considered a fundamental attribute of dinoflagellate life cycles [19], that in M. polykrikoides is also a research "hot" topic. The ability of M. polykrikoides to produce resting cysts in culture was first confirmed in 2012, and possible ecological implication was then discussed, such as the contribution to bloom expansion and annual recurrence [20]. M. polykrikoides is also able to form hyaline cysts, which are immotile chain-forming cells surrounded by a transparent membrane [21]. However, there is little report about resting cysts in $M$. fulvescens in previous studies.

Among species of the genus Margalefidinium, M. fulvescens and M. polykrikoides may often coexist in some coastal waters [22,23]. M. fulvescens and M. polykrikoides are not easily distinguished morphologically under LM due to their similar morphological characteristics, especially after preservation [5,7]. In addition, the microscopic quantification of Margalefidinium species at lower abundance is highly challenging, particularly in the case of post-preservation identification, necessitating alternative approaches such as molecular tools [7]. In recent years, molecular techniques used to identify genetic diversity and provide new insight into the physiology and ecology of phytoplankton. Through monoclonal isolation and partial regions of large subunit ribosomal RNA gene (LSU rDNA) sequences, researchers divided M. polykrikoides strains into four clades (i.e., ribotypes): East Asian, Philippines, American/Malaysian, and Mediterranean type $[8,9,17]$. With the advancement of next-generation sequencing technologies, metabarcoding analysis has been demonstrated to have great potential to strengthen biological monitoring phytoplankton communities in the seawaters. Hattenrath-Lehmann and colleagues [24] conducted metabarcoding analyses using partial 16S rRNA (V4 region) and 18S rRNA (V7-8 regions) during M. polykrikoides bloom in USA, and found that the lower diversity of plankton communities of blooms might be due to allelopathically excluded by M. polykrikoides. Cui and colleagues studied microbial module regulations on the M. polykrikoides blooms using 
$16 \mathrm{~S}$ rRNA (V3-V5 region for Archaea; V3-V4 for Bacteria) and 18S rRNA (V8-9 regions) as molecular markers [25].

Margalefidinium species only recently emerged as the dominant HAB species after persisting for years at low abundance of the total phytoplankton communities. For example, the first outbreak of M. polykrikoides in Korea was in 1981, and since then, M. polykrikoides blooms occurred regularly and expanded to most of the country's coastal areas $[9,26]$. Similar phenomena were also found in the coastal waters of California, USA [12], and the Mediterranean Sea [10]. Thus, it was urgent to track these species distributions before their blooms outbreak, especially for the seawaters without previously recorded Margalefidinium blooms. Despite extensive research on Margalefidinium species, the biodiversity and spatialtemporal dynamics of these species remain obscure. Knowledge of biodiversity and spatial-temporal dynamics may shed light on the understanding of abrupt occurrences and continuity of Margalefidinium blooms in ocean regions globally.

Jiaozhou Bay, which is a semi-enclosed bay with an area of approximately $367 \mathrm{~km}^{2}$, located on the western coast of the Yellow Sea [27], is an excellent ocean region for carrying out research on the biodiversity and spatial-temporal dynamics of Margalefidinium species. M. polykrikoides and M. fulvescens have been described in Jiaozhou Bay $[5,28]$. On the basis of analysis of large subunit (LSU) sequences, M. polykrikoides in Jiaozhou Bay belongs to the East Asian ribotype [28], and M. polykrikoides of Jiaozhou Bay strains (MPJZB-C3 and MPJZB-D6) showed lower toxicity than that of USA strain (CP1) and Malaysia strain (MPCoKK23) [16]. Hu and colleagues [5] confirmed the presence of M. fulvescens in Jiaozhou Bay through morphological and phylogenetic characterization, and found that M. fulvescens dominated the dinoflagellate community of Jiaozhou Bay in the early autumn of 2015. Recently, Lin and colleagues [29] assessed the intra-populational and intra-genome genetic diversity of $M$. fulvescens and inferred high variability among LSU copies. Although no severe HABs caused by Margalefidinium species in Jiaozhou Bay has been reported [30], the potential of Margalefidinium bloom outbreaks is high. Thus, it is important to detect these Margalefidinium species in Jiaozhou Bay.

In this study, we conducted metabarcoding analysis using $18 \mathrm{~S}$ rDNA V4 regions for samples collected monthly at 12 sites in Jiaozhou Bay in 2019, targeting Margalefidinium species. We confirmed the presence of M. fulvescens and M. polykrikoides, and the two species showed high genetic diversity (may be copy diversity in the individual cells). We also compared the spatial-temporal dynamics of Margalefidinium species, as well as the environmental factors.

\section{Materials and Methods}

\subsection{Sampling Collection}

We conducted 12 monthly expedition voyages in 2019 in Jiaozhou Bay, sampling at 12 sites during each expedition (Figure 1). At each site, $1 \mathrm{~L}$ of seawater was collected at different sampling depths. The number of samples collected at each sampling site depended on the water depth of the corresponding sampling site. Water filtration and storage were the same as described previously [31]. Briefly, each seawater sample was filtered using a $200 \mu \mathrm{m}$ mesh (Hebei Anping Wire Mesh Co., Ltd., Hengshui, China) to remove large phytoplankton and debris, followed by filtration using $10 \mu \mathrm{m}$ polycarbonate membranes (Millipore, Burlington, MA, USA) and $0.2 \mu \mathrm{m}$ polycarbonate membranes, sequentially. The membranes with samples were stored in liquid nitrogen until they are used for DNA exactions. A total of 468 filtered membrane samples were obtained in this study. 


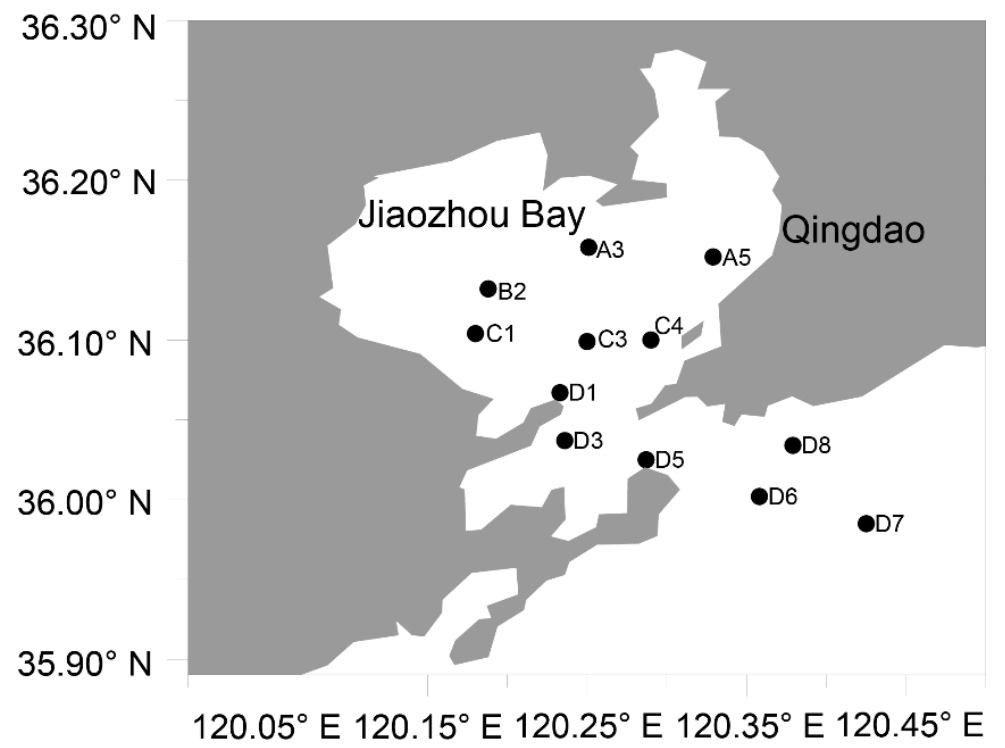

Figure 1. Sampling sites in Jiaozhou Bay.

Environmental data (temperature, salinity, $\mathrm{SiO}_{3}{ }^{2-}, \mathrm{PO}_{4}{ }^{3-}, \mathrm{NO}_{2}{ }^{-}, \mathrm{NO}_{3}{ }^{-}, \mathrm{NH}_{4}{ }^{+}$, and chlorophyll concentrations) were provided by Jiaozhou Bay National Marine Ecosystem Research Station, Institute of Oceanology, Chinese Academy of Sciences.

\subsection{DNA Extraction, PCR Amplification, and Sequencing}

For each membrane with filtrated samples, $500 \mu \mathrm{L}$ CSPL buffer (Omega, Norwalk, CT, USA) was quickly added after they were taken out of liquid nitrogen. It was then cut into pieces using sterilized scissors for approximately 50 times, followed by crushing in a cell crushing apparatus (MP Biomedicals, Santa Ana, USA) for $5 \mathrm{~s}$ at a speed of $4 \mathrm{~m} / \mathrm{s}$. DNA was subsequently extracted using the HP Plant DNA Kit (Omega, Norwalk, CT, USA), according to the manufacturer's instructions.

The $18 \mathrm{~S}$ rDNA v4 regions were amplified using the V4F forward primer, CCAGCA (G/C)C(C/T)GCGGTAATTCC, and the V4R reverse primer, ACTTTCGTTCTTGAT(C/T) (A/G)A [32], with a unique barcode to distinguish different samples. Each PCR amplification was performed in a final volume of $50 \mu \mathrm{L}$, including $50 \mathrm{ng}$ template DNA (n $\mu \mathrm{L})$, $25 \mu \mathrm{L} 2 X$ Taq PCR Master Mix (Tiangen Biotech, Beijing, China), $1 \mu \mathrm{L}$ forward primer, and $1 \mu \mathrm{L}$ reverse primer. The remaining volume (23-n $\mu \mathrm{L}$ ) was supplemented with $\mathrm{ddH}_{2} \mathrm{O}$ to reach a total of $50 \mu \mathrm{L}$. PCR cycling conditions included an initial denaturation at $94{ }^{\circ} \mathrm{C}$ for $4 \mathrm{~min}$, followed by 32 cycles of denaturation at $94^{\circ} \mathrm{C}$ for $1 \mathrm{~min}$, annealing at $57^{\circ} \mathrm{C}$ for $1 \mathrm{~min}$ and $50 \mathrm{~s}$, elongation at $72{ }^{\circ} \mathrm{C}$ for $2 \mathrm{~min}$, and a final extension at $72{ }^{\circ} \mathrm{C}$ for $10 \mathrm{~min}$. To check the product length, PCR products were monitored on $1 \%$ agarose gels, followed by purification with the Qiagen Gel Extraction Kit (Qiagen, Germany). Sequencing libraries were generated using the TruSeq ${ }^{\circledR}$ DNA PCR-Free Sample Preparation Kit (Illumina, Inc., San Diego, CA, USA). Library quality was assessed on a Qubit@ 2.0 Fluorometer (Thermo Scientific, Waltham, MA, USA) and the Agilent Bioanalyzer 2100 system (Agilent Technologies, Santa Clara, CA, USA). Sequencing was conducted on the Illumina NovaSeq platform (Novogene, Beijing, China) with 250 bp paired-end reads. Raw data have been deposited to NCBI under the BioProject number PRJNA577777 and PRJNA733859.

\subsection{Bioinformatics and Statistical Analyses}

DADA2 analysis was conducted in $\mathrm{R}[33,34]$, and the parameters were set as follows: $\operatorname{maxEE}=c(2,2)$, minLen $=200$, truncLen $=c(220,220)$, $\operatorname{minBoot}=80$, and Min overlap $=12$ bases. To maintain the same sequencing depths, we subsampled the number of reads per sample to the lowest number of reads per samples $(48,286)$. In this study, amplicon sequence variants (ASVs) supported by at least two reads were retained for further 
analysis. Annotation was done in two steps. First, the initial taxonomical assignment was performed according to the Protist Ribosomal Reference (PR2) database [35]. We assigned taxonomy up to the genus level with the assignTaxonomy function at an $80 \%$ bootstrap confidence threshold. Second, further taxonomic assignment was done for all ASVs that were not annotated to any species at the first step through using BLAST against the NCBI NT database. Each ASV was annotated to the species in the reference database with the highest percentage identity (PID) at a threshold of 99\%. For the species level annotation, all ASVs that were annotated to multiple species were removed.

To study the spatial and temporal distribution of Margalefidinium species in Jiaozhou Bay, we extracted 28 ASVs annotated as Margalefidinium. After subsampling, each sample contained 48,286 reads. The relative abundance of each ASV was represented by the number of reads in each sample. Phylogenetic trees of Margalefidinium ASVs were generated with MEGAX [36] using the maximum likelihood (ML) method and Kimura 2-parameter model with 1000 bootstrap. Surfer 16 (Golden Software LLC, Golden, CO, USA) was used to illustrate sampling sites and spatial distribution of ASV_38. The TCS network of Margalefidinium ASVs was constructed by PopART v1.7 [37,38]. Correlation analysis between ASVs and environmental factors was carried out using spearman correlation in the R package corrplot [39]. Bubble charts were drawn with the R package ggplot2 [40]. Line plots were drawn using GraphPad Prism v8 (GraphPad Software, San Diego, CA, USA).

\section{Results}

\subsection{Biological Diversity of Margalefidinium Species in Jiaozhou Bay}

A total of 45,932 ASVs were obtained from the seawater samples collected from the 12 sampling sites in Jiaozhou Bay (Figure 1) in 12 months in 2019, 30,097 ASVs of which were supported by at least two reads [33]. Of these 30,097 ASVs, 28 ASVs were annotated as Margalefidinium species, including 1 ASV corresponding to M. polykrikoides, 18 ASVs corresponding to M. fulvescens, and 9 ASVs annotated to an unknown species of the genus Margalefidinium (Table 1). Eighteen distinct ASVs were annotated as M. fulvescens, suggesting that this species had high intra-species genetic diversity. Among these ASVs annotated as M. fulvescens, ASV_38 showed the highest abundance. Both M. polykrikoides and $M$. fulvescens were both annotated as HAB species $[5,16]$ and both have been reported previously in Jiaozhou Bay [5,28].

Table 1. List of ASVs annotated as Margalefidinium species detected in this study in Jiaozhou Bay.

\begin{tabular}{clc}
\hline ASV_Name & \multicolumn{1}{c}{ Species } & Group \\
\hline ASV_38 & Margalefidinium fulvescens, AB288380,100 & mvs1 \\
ASV_1321 & Margalefidinium fulvescens, AB288380,99.732 & mvs1 \\
ASV_1350 & Margalefidinium fulvescens, AB288380,99.464 & mvs1 \\
ASV_2308 & Margalefidinium fulvescens, AB288380,99.732 & mvs1 \\
ASV_2479 & Margalefidinium fulvescens, AB288380,99.732 & mvs1 \\
ASV_3857 & Margalefidinium fulvescens, AB288380,99.732 & mvs1 \\
ASV_4106 & Margalefidinium fulvescens, AB288380,99.732 & mvs1 \\
ASV_5956 & Margalefidinium fulvescens, AB288380,99.464 & mvs1 \\
ASV_7153 & Margalefidinium fulvescens, AB288380,99.732 & $\mathrm{mvs1}$ \\
ASV_7377 & Margalefidinium fulvescens, AB288380,99.732 & $\mathrm{mvs1}$ \\
ASV_7383 & Margalefidinium fulvescens, AB288380,99.732 & $\mathrm{mvs1}$ \\
ASV_7789 & Margalefidinium fulvescens, AB288380,99.732 & $\mathrm{mvs1}$ \\
ASV_8950 & Margalefidinium fulvescens, AB288380,99.732 & $\mathrm{mvs1}$ \\
ASV_9769 & Margalefidinium fulvescens, AB288380,99.464 & $\mathrm{mvs1}$ \\
ASV_10236 & Margalefidinium fulvescens, AB288380,99.464 & $\mathrm{mvs1}$ \\
ASV_11070 & Margalefidinium fulvescens, AB288380,99.732 & $\mathrm{mvs1}$ \\
ASV_13005 & Margalefidinium fulvescens, AB288380,99.732 & $\mathrm{mvs1}$ \\
ASV_25960 & Margalefidinium fulvescens, AB288380,99.464 & $\mathrm{mvs1}$ \\
ASV_1149 & Margalefidinium polykrikoides, AY347309,100 & $1 \mathrm{vs1}$ \\
\hline
\end{tabular}


Table 1. Cont.

\begin{tabular}{cll}
\hline ASV_Name & Species & Group \\
\hline ASV_1311 & Margalefidinium sp. & genus level \\
ASV_6970 & Margalefidinium sp. & genus level \\
ASV_11371 & Margalefidinium sp. & genus level \\
ASV_11381 & Margalefidinium sp. & genus level \\
ASV_12769 & Margalefidinium sp. & genus level \\
ASV_13519 & Margalefidinium sp. & genus level \\
ASV_14867 & Margalefidinium sp. & genus level \\
ASV_16662 & Margalefidinium sp. & genus level \\
ASV_19458 & Margalefidinium $\mathrm{sp.}$ & genus level \\
\hline
\end{tabular}

\subsection{Spatial-Temporal Dynamics of Margalefidinium Species in Jiaozhou Bay}

We found that different Margalefidinium species have quite different distribution patterns in Jiaozhou Bay. Furthermore, different ASVs of the same species also displayed different spatial-temporal dynamics. The relative abundance revealed that Margalefidinium species have obvious seasonal distribution characteristics. They were mostly detected in early autumn, especially in September, suggesting that Margalefidinium species preferred a relatively higher temperature (Figure 2). In terms of geographical distribution, it showed little preference among the 12 sampling sites (Figure 3). In addition, the spatial-temporal dynamics of Margalefidinium species were consistent in two different seawater samples in $10-200 \mu \mathrm{m}$ and in $0.2-10 \mu \mathrm{m}$.
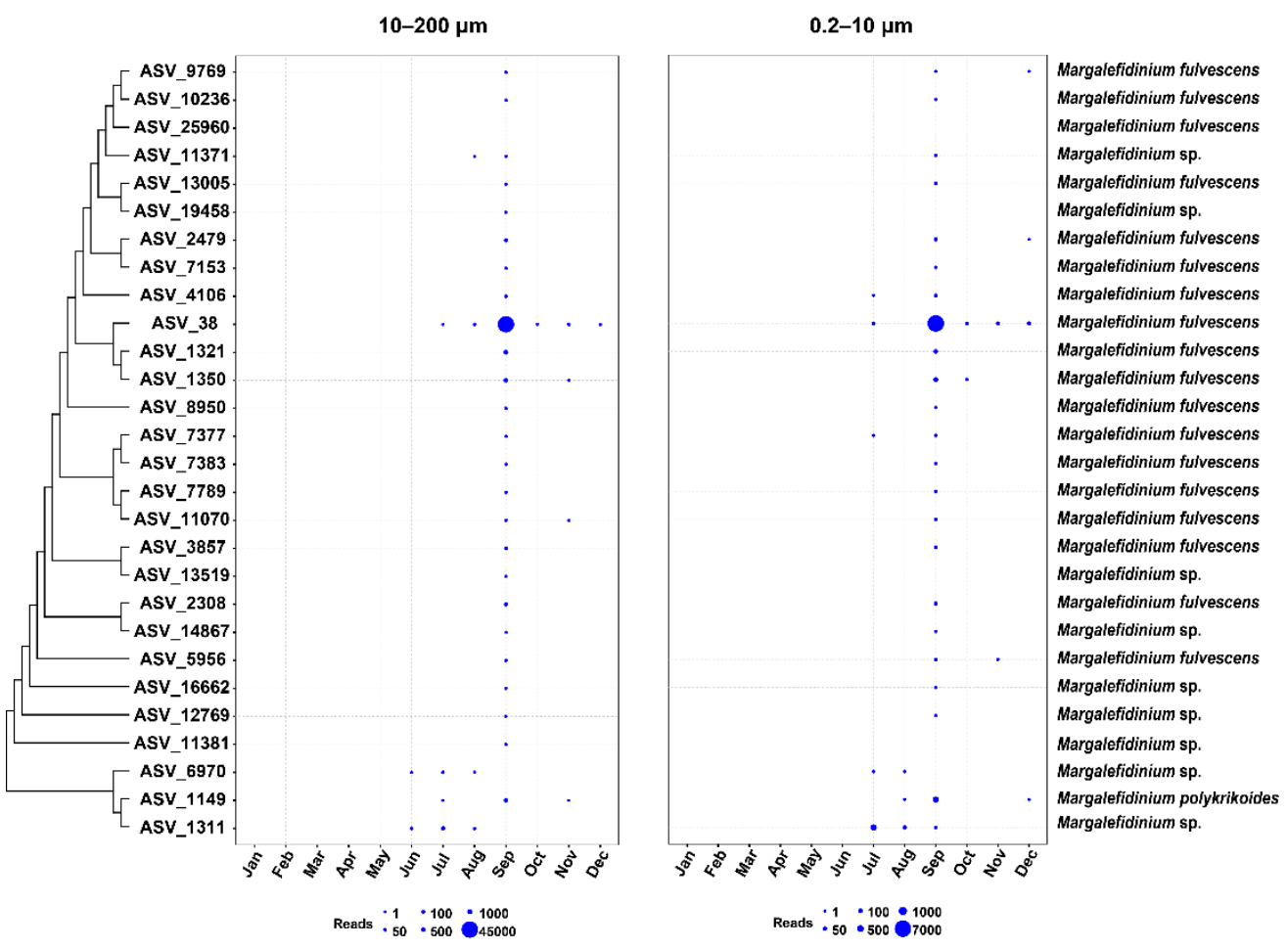

Figure 2. Temporal dynamics of Margalefidinium species for the surface water in Jiaozhou Bay.

For further analysis, ASV_38, with the highest abundance among Margalefidinium species, was selected to study the regularities of distribution. It was annotated as $M$. fulvescens and was detected in the seawater samples from July to December (Figure 4a). From July to August, ASV_38 was found only outside the bay, and the number was increasing. By September, it had the largest quantity and distributed in all 12 sampling sites in Jiaozhou Bay. From October to December, the relative abundance of ASV_38 was decreasing, but it was found both inside and outside the bay. 

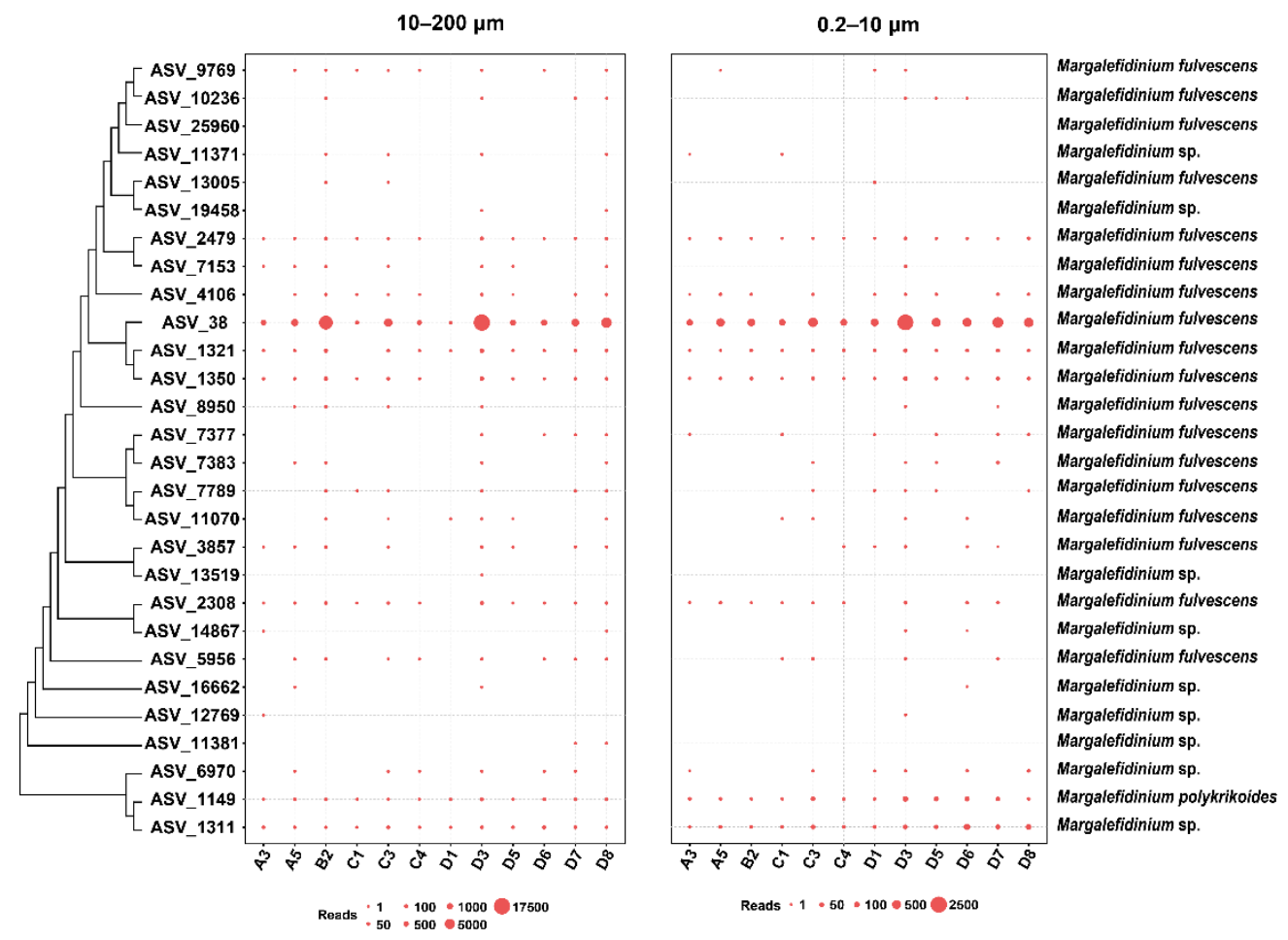

Figure 3. Spatial variations of Margalefidinium species for the surface water in Jiaozhou Bay.

We further analyzed the vertical distribution profiles of ASV_38 (M. fulvescens) at different seawater depths in September, considering the strong vertical migration capability of dinoflagellates [41]. In general, the ASV_38 values were highest or close to the highest at the surface (Figure 4), and the minimum values were found at the bottom with a few exceptions (Figure $4 \mathrm{~b}$ ). At sites C3 and D7, wherein both had similar seawater depths (about $15 \mathrm{~m}$ ), ASV_38 reached maximum values at the surface. However, the minimum values of these two samples sites were different. While the minimum value was found at $10 \mathrm{~m}$ water under the surface at the $\mathrm{C} 3$ site, the minimum value was found at the bottom at the D7 site. At the D6 site, which was located outside of Jiaozhou Bay, the seawater depth reached $25 \mathrm{~m}$. It also had the maximum of ASV_38 reads at the surface of seawater and the minimum on the bottom, similar to site D7. However, the difference between the bottom and middle layers was substantially reduced compared to the D7 site. The D5 site had the deepest depth among all 12 sampling sites in this expedition in Jiaozhou Bay $(\approx 30 \mathrm{~m})$ and had the unique distribution pattern of ASV_38. From the surface to the bottom, the number of reads decreased first, then increased to the maximum, and finally decreased to the minimum.

\subsection{Substantial Genetic Diversity of Margalefidinium Species in Jiaozhou Bay}

The phylogenetic tree indicated that the 28 ASVs uncovered in the DADA2 analysis formed two major groups (Figure 5a). While most ASVs were annotated to be M. polykrikoides or M. fulvescens, some were annotated as Margalefidinium sp. These ASVs were substantially different from reference $18 \mathrm{~S}$ rDNA V4 region sequences of these two Margalefidinium species. Nevertheless, these ASVs annotated as Margalefidinium sp. were clustered closely with M. polykrikoides or M. fulvescens. The ASV_11381 and ASV_12769 might have a closer relationship with M. fulvescens. The ASV_6970 and ASV_1311 seemed to have a closer relationship with $M$. polykrikoides. The phylogenetic network displayed the same results in a different format, highlighting relative abundance of each ASV (Figure $5 b$ ). ASV_38 (M. fulvescens) was the most abundant haplotype, with most other haplotypes surrounding it, suggesting that many strains with highly similar haplotypes. The ASV_1149, representing M. polykrikoides, together with ASV_1311 and ASV_6970, formed the other 
group. The ASV_6970, which played an important role in connecting the two groups, revealed that these two groups had many sites that are different from each other.

a
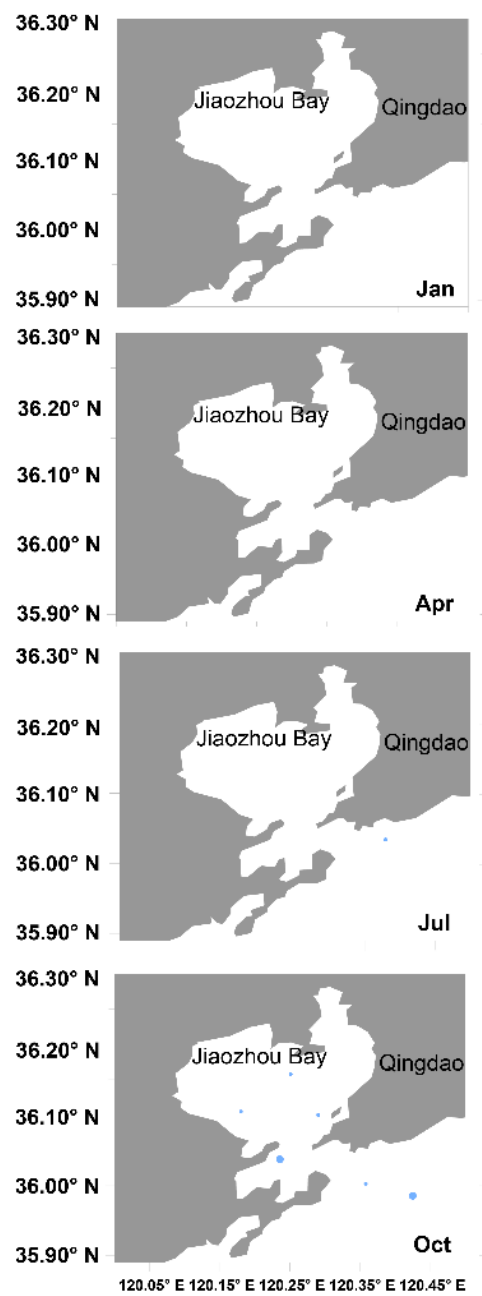

Oct

$120.05^{\circ} \mathrm{E} 120.15^{\circ} \mathrm{E} 120.25^{\circ} \mathrm{E} 120.35^{\circ} \mathrm{E} 120.45^{\circ} \mathrm{E}$
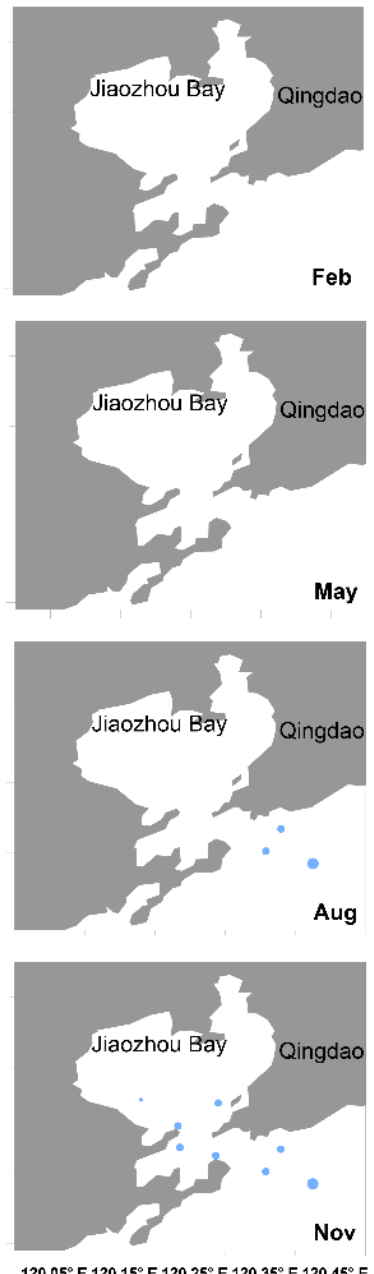

Nov

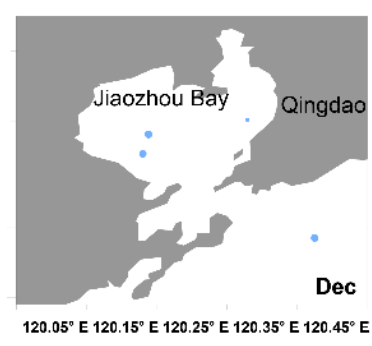

$120.05^{\circ} \mathrm{E} 120.15^{\circ} \mathrm{E} 120.25^{\circ} \mathrm{E} 120.35^{\circ} \mathrm{E} 120.45^{\circ} \mathrm{E}$
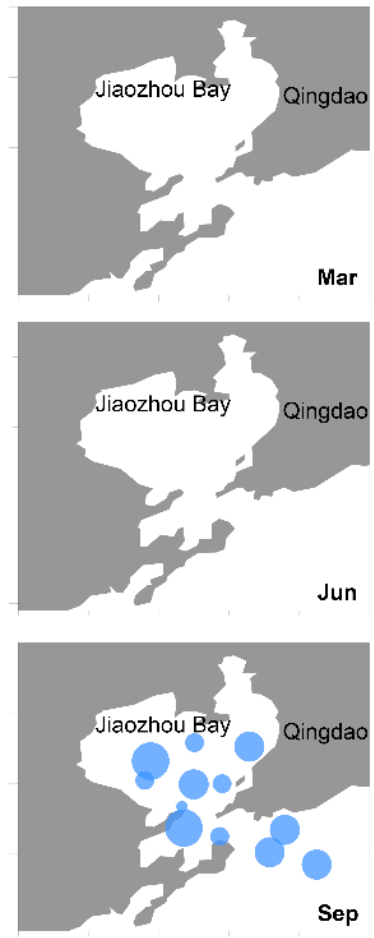

b
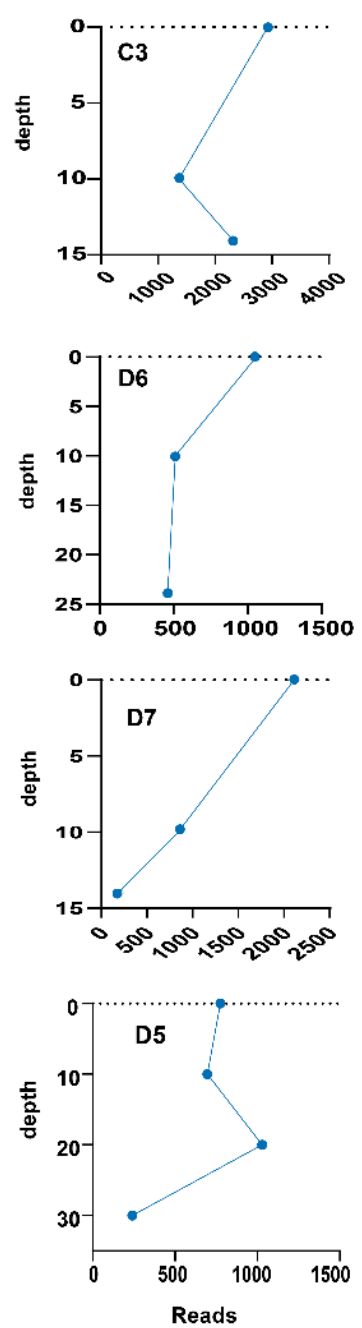

Reads

1 to 2 - 2 to $10 \quad 10$ to 50 50 to $100 \quad 100$ to 1000

1000 to 10000

10000 to 20000

Figure 4. The spatial and temporal distributions of M. fulvescens (ASV_38). (a) Horizontal distributions of M. fulvescens for 12 months in 10-200 $\mu \mathrm{m}$ fraction of surface water in Jiaozhou Bay. (b) The vertical distribution of M. fulvescens in September for C3, D6, D7, and D5 sites.

\subsection{Correlation of Margalefidinium Species with Environmental Factors}

To explore the impact of environmental factors on the temporal time course of Margalefidinium species, we calculated correlation coefficients between relative abundance of ASVs and environmental variables in Jiaozhou Bay (Figure 6a). It was found that Margalefidinium species were positively correlated with temperature. Some of these ASVs were significantly positively correlated with temperature, indicating that temperature was the most important influencing factor for Margalefidinium species (Figure 6b). All ASVs showed negative correlations with $\mathrm{SiO}_{3}{ }^{2-}$, and, in particular, ASV_38 (M. fulvescens) was significantly negatively correlated with $\mathrm{SiO}_{3}{ }^{2-}$ (Figure 6a). Furthermore, all ASVs showed positive correlations with $\mathrm{NH}_{4}{ }^{+}$, and ASV_38 (M. fulvescens) showed significant positive correlation with $\mathrm{NH}_{4}{ }^{+}$(Figure 6c). Margalefidinium species did not have a significant correlation with other environmental factors, suggesting that Margalefidinium species 
may have strong ecological adaptability and a wide distribution, consistent with previous studies $[16,42]$.
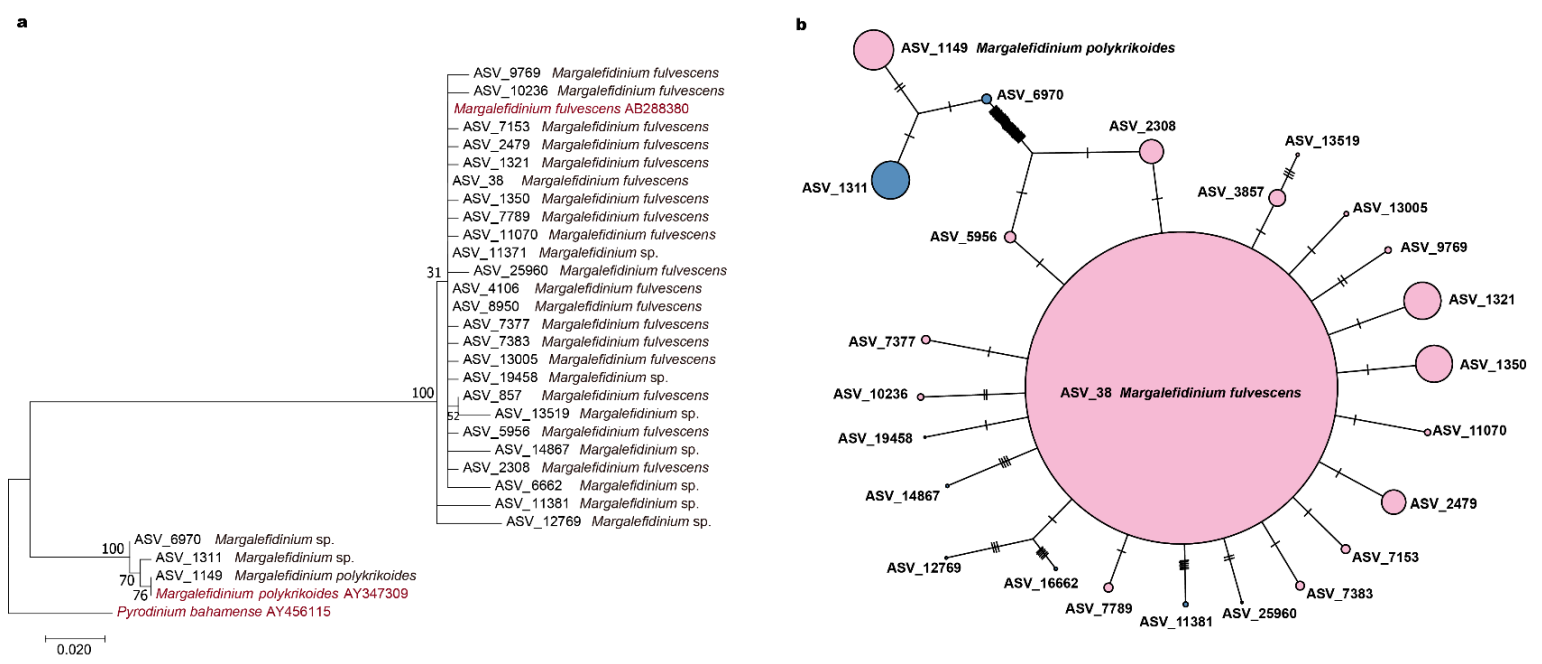

Figure 5. The phylogenetic tree (a) and TCS network of Margalefidinium species in Jiaozhou Bay (b). The pink points refer to the ASVs annotated as being M. fulvescens or M. polykrikoides and the blue points refer to the ASVs annotated as being from the Margalefidinium genus. The size of dots is in correspondence to the relative abundance of Margalefidinium ASV in Jiaozhou Bay.

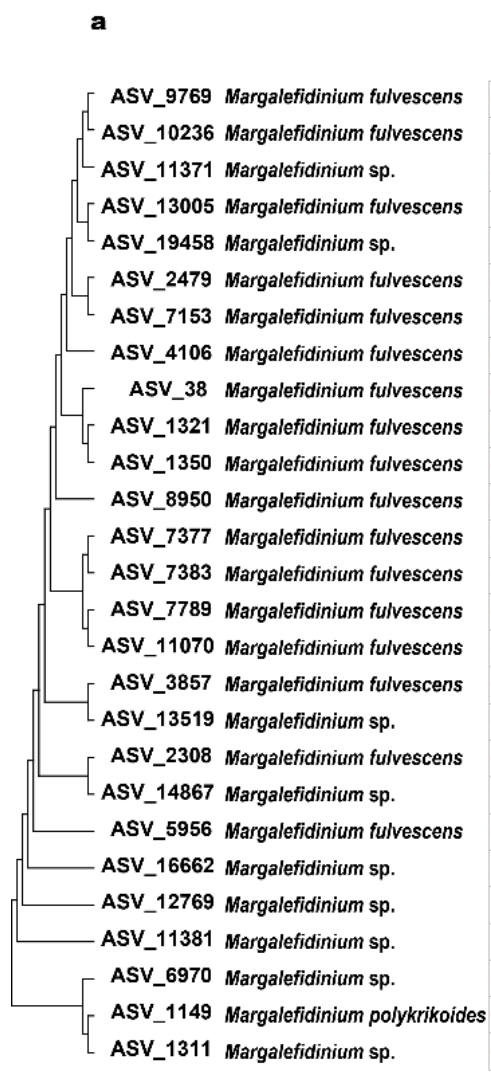

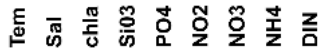

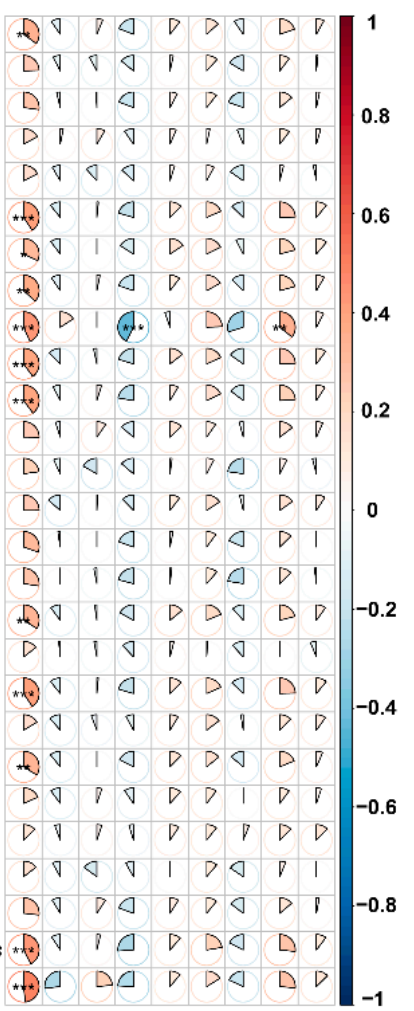

b

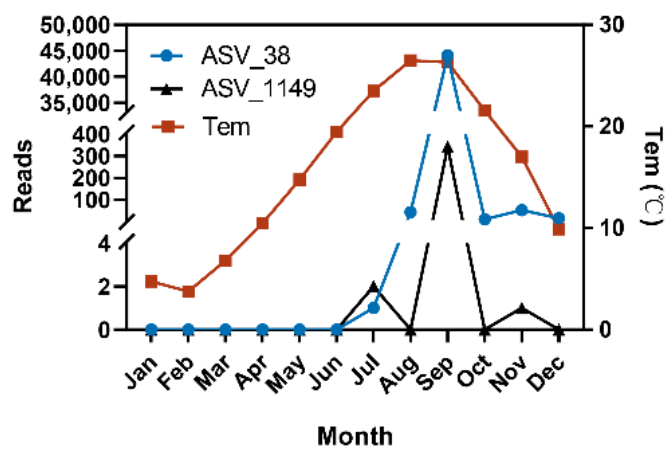

c

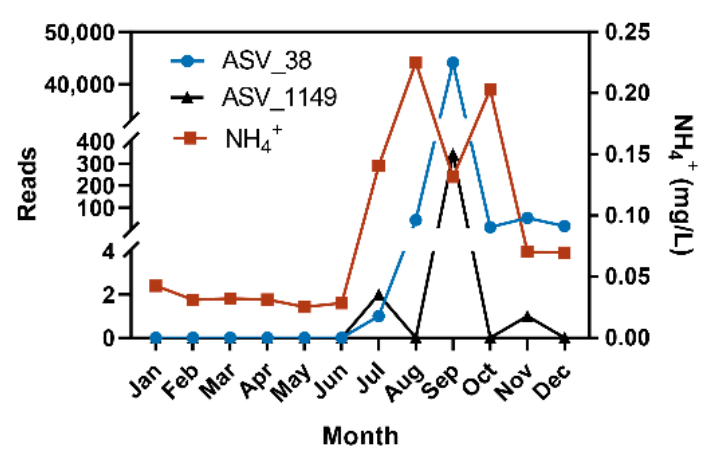

Figure 6. (a) Correlation of Margalefidinium species with environmental factors in 10-200 $\mu \mathrm{m}$ fraction of the surface water in Jiaozhou Bay. The pies with * indicate that $p$-value $<0.05$, the pies with ${ }^{* *}$ indicate $p$-value $<0.01$, the pies with ${ }^{* * *}$ indicate $p$-value $<0.001$. (b) Temporal variations of ASV_38 (M. fulvescens) abundances and ASV_1149 (M. polykrikoides) abundances, and temperature for the 10-200 $\mu \mathrm{m}$ fraction for the surface water in Jiaozhou Bay. (c) Temporal variations of ASV_38 (M. fulvescens) abundances and ASV_1149 (M. polykridoides) abundances, and $\mathrm{NH}_{4}{ }^{+}$concentrations for the 10-200 $\mu \mathrm{m}$ fraction for the surface water in Jiaozhou Bay. 


\section{Discussion}

High-throughput metabarcoding is now routine for studying protist diversity and distributions, having been applied in multitude environments at different sampling scales [43], for example, Tara Oceans Expedition [44] and the Ocean Sampling Day [45]. This method provides new opportunities in studying protist diversity, especially for these rare, small, or cryptic species [46]. For time series samples, metabarcoding analysis has also been ongoing for marine investigation, such as in the Mediterranean coast [47]. Thus, metabarcoding analysis could be used for routinely monitoring the HAB species.

Despite extensive research on Margalefidinium species, many of which are cosmopolitan $\mathrm{HAB}$ species with negative impact on economy and ecosystems, the biodiversity and spatial-temporal dynamics of these species remain poorly understood. Through carrying out metabarcoding analysis of samples collected in Jiaozhou Bay (China) for one entire year, we gained quantitative insight into the spatial-temporal dynamics of Margalefidinium species.

\subsection{Low Biodiversity of Margalefidinium Species but High Intra-Species Genetic Diversity Was Identified in Jiaozhou Bay}

According to current taxonomy system, the genus Margalefidinium is relatively small, only containing five species [1]. Two species, M. polykrikoides and M. fulvescens, have been detected in coastal regions in China [23]. In our project, we successfully identified these two species among the 28 ASVs corresponding to species in the genus Margalefidinium in Jiaozhou Bay. According to phylogenetic analysis based on $18 \mathrm{~S}$ rDNA V4 region (Figure 5), these two species formed two sister groups, which was confirmed by previous studies that constructed a phylogenetic tree based on $28 \mathrm{~S}$ rDNA [17]. Moreover, there were nine ASVs annotated to the genus level; however, they were clustered together with M. polykrikoides and $M$. fulvescens instead of forming a separate clade due to larger than $1 \%$ nucleotide differences compared with reference sequences in NCBI (Table 1, Figure 5). The annotation result, as well as the phylogenetic analysis, indicated the low biodiversity of Margalefidinium species in Jiaozhou Bay.

Interestingly, high intra-species genetic diversity was found in Margalefidinium species. Previous studies have proven that the different geographic populations of M. polykrikoides around the world are in fact genetically different according to the $28 \mathrm{~S}$ rDNA gene, and thus they were divided into four ribotypes: the East Asian ribotype, the American/Malaysian ribotype, the Philippines ribotype, and the Mediterranean ribotype $[17,28]$. In this study, only one ASV (ASV_1149) was specifically annotated as M. polykrikoides on the basis of 18S rDNA V4, and two suspected ASVs clustered with M. polykrikoides (ASV_1311 and ASV_6970) were annotated as Margalefidinium sp. A similar discovery was made in the American/Malaysian ribotype in that despite the genetic similarity of the 28S rDNA gene of clones within the ribotype, there may be ecological differences among these populations [7]. We found 25 ASVs clustered with M. fulvescens according to 18S rDNA V4 (Figure 5), and among them, 18 ASVs were annotated as M. fulvescens in Jiaozhou Bay. Thus, it was hypothesized that $M$. fulvescens could also be divided into multiple ribotypes, which still need to be confirmed [7]. However, it could not be ruled out that diversities among these 25 ASVs might be due to high copy numbers of ribosomal gene clusters within individual cells of $M$. fulvescens, which was estimated to up to 5620 copies per cell by using the correlation between cell length and rDNA copy number [48]. The study based on $28 \mathrm{~S}$ rDNA analysis inferred that the variability within individual cells (i.e., variability among polymorphic copies of $28 \mathrm{~S}$ rDNA) caused both the intra-individual and intrapopulational genetic diversities rather than the co-existence of populations of different geographic origins. 
4.2. Margalefidinium Species in Jiaozhou Bay Demonstrated Strong Temporal Dynamics with a Sharp Peak of Abundance in Early Autumn

In our research of Jiaozhou Bay in 2019, the relative abundance of Margalefidinium species demonstrated strong temporal dynamics with a sharp peak of abundance in early autumn (Figure 2). In contrast, the relative abundance of Margalefidinium species showed no obvious spatial preference (Figure 3). These results were congruent with a previous study from August to October in 2015 in Jiaozhou Bay, reaching 68,000 cells/L for the total density of M. polykrikoides and M. fulvescens in the early autumn [5]. Further study found that Margalefidinium species showed the highest correlation with water temperature among different environmental factors in this study (Figure 6). The species M. polykrikoides have been proven to be eurythermal, well adapted to warm $\left(>20^{\circ} \mathrm{C}\right)$ offshore, possibly tropical or subtropical waters $[49,50]$. The optimum temperature for growth is relatively high, ranging from 23 to $27^{\circ} \mathrm{C}$, although it can remain viable down to temperatures of approximately $12.5^{\circ} \mathrm{C}$ [51]. Distinct from M. polykrikoides, M. fulvescens appears to be adapted to relatively lower temperatures, associated with cooler coastal upwelling conditions according to the extremely limited data available [7,13]. In Jiaozhou Bay in 2019, the water temperature from January to May ranged between 3 and $17^{\circ} \mathrm{C}$, being neither friendly to the growth of M. polykrikoides nor $M$. fulvescens, and therefore we failed to detect them. In summer to early autumn (from June to September), the water temperature rose, and the maximum reached above $26^{\circ} \mathrm{C}$, being consistent with high abundance of two Margalefidinium species, especially M. fulvescens (ASV_38). The relatively higher abundance of M. fulvescens compared to M. polykrikoides was consistent with the previous observation, in which M. fulvescens appeared to be adapted to lower temperature than M. polykrikoides according to the current study [7]. On the other hand, the copy number differences of rDNA per individual cells might be another reason. Our results confirmed that water temperature is a crucial factor for the abundance of Margalefidinium species.

Both M. polykrikoides and M. fulvescens are euryhaline, well adapted to moderate (30-33) salinities sea waters $[49,50]$. In Jiaozhou Bay in 2019, there was little change in salinity among the months (31.08-32.03), and thus it may not be an explanation for the sharp peak of abundance of Margalefidinium species in early autumn.

Margalefidinium species exhibits wide flexibility in its nutrient acquisition strategies [7]. Previous studies found that they have a preference for $\mathrm{NH}_{4}{ }^{+}$versus $\mathrm{NO}_{2}{ }^{-}$and $\mathrm{NO}_{3}{ }^{-}$[7], and in our research, we obtained similar results. All ASVs showed positive correlations with $\mathrm{NH}_{4}{ }^{+}$concentration in Jiaozhou Bay, and ASV_38 (M. fulvescens) showed a significantly positive correlation. In August 2019, before the appearance of the peak abundance of Margalefidinium species, the concentration of $\mathrm{NH}_{4}{ }^{+}$maximized, which may significantly contribute to the growth of Margalefidinium species. Then, in September, when abundance of Margalefidinium species went to the maximum, $\mathrm{NH}_{4}{ }^{+}$was obviously consumed, lower than the neighbor months. As a result, we inferred that the concentration of $\mathrm{NH}_{4}{ }^{+}$is a possible factor for the sharp peak of abundance of Margalefidinium species in early autumn. Furthermore, it was supported by previous reviews that both M. polykrikoides and M. fulvescens can readily utilize organic nitrogen and phosphorus compounds [7], which may explain the result found in our study that Margalefidinium species do not have significant correlation with some of the environmental factors, such as $\mathrm{NO}_{2}{ }^{-}$and $\mathrm{NO}_{3}{ }^{-}$. Thus, we next need to pay more attention to the organic substance carried into Jiaozhou Bay instead of the inorganic nutrient to better explain the sharp peak of abundance of Margalefidinium species in early autumn.

\section{Conclusions}

Our data provided the first analysis of monthly and spatial dynamics of Margalefidinium species in Jiaozhou Bay using the metabarcoding approach. In this study, we detected two Margalefidinium species (M. polykrikoides and M. fulvescens) with high genetic diversity in Jiaozhou Bay. M. polykrikoides and M. fulvescens showed a strong temporal shift with a sharp peak of abundance in early autumn around the whole year, while they 
did not show significant preference among different sites in Jiaozhou Bay. Our results also suggested the temperature might be the main contributor for their obvious temporal dynamics. Thus, the summer and early autumn might be the possible outbreak time of Margalefidinium species blooms in Jiaozhou Bay, which requires more attention.

Author Contributions: N.C. conceived of the study; S.L. performed the experiments; M.Z., S.L., and N.C. analyzed the data and wrote the paper; Y.Z. offered the environmental factors. All authors have read and agreed to the published version of the manuscript.

Funding: This research was funded by the Natural Science Foundation of China (41906118), the Strategic Priority Research Program of Chinese Academy of Sciences (XDB42000000), the Chinese Academy of Sciences Pioneer Hundred Talents Program (to Nansheng Chen), the Taishan Scholar Project Special Fund (to Nansheng Chen), the Qingdao Innovation and Creation Plan (Talent Development Program - 5th Annual Pioneer and Innovator Leadership Award to Nansheng Chen, 19-3-2-16-zhc), and the National Key Research and Development Program of China (2017YFC1404300).

Institutional Review Board Statement: Not applicable.

Informed Consent Statement: Not applicable.

Data Availability Statement: The sequencing results (raw data) have been submitted to NCBI, and the BioProject numbers are PRJNA577777 and PRJNA733859.

Acknowledgments: We are grateful to colleagues from the Jiaozhou Bay Marine Ecosystem Research Station for the opportunity to participate in the investigation expeditions and for their help with field sampling.

Conflicts of Interest: The authors declare that they have no conflict of interest.

\section{References}

1. Gomez, F.; Richlen, M.L.; Anderson, D.M. Molecular characterization and morphology of Cochlodinium strangulatum, the type species of Cochlodinium, and Margalefidinium gen. nov. for C. polykrikoides and allied species (Gymnodiniales, Dinophyceae). Harmful Algae 2017, 63, 32-44. [CrossRef]

2. Guiry, M.D.; Guiry, G.M. AlgaeBase. 2021. Available online: https://www.algaebase.org (accessed on 31 August 2021).

3. Al-Azri, A.R.; Al-Hashmi, K.A.; Al-Habsi, H.; Al-Azri, N.; Al-Khusaibi, S. Abundance of harmful algal blooms in the coastal waters of Oman: 2006-2011. Aquat. Ecosyst. Health 2015, 18, 269-281. [CrossRef]

4. Iwataki, M.; Kawami, H.; Matsuoka, K. Cochlodinium fulvescens sp. nov. (Gymnodiniales, Dinophyceae), a new chain-forming unarmored dinoflagellate from Asian coasts. Phycol. Res. 2007, 55, 231-239. [CrossRef]

5. Hu, Z.; Deng, Y.; Li, Y.; Tang, Y.Z. The morphological and phylogenetic characterization for the dinoflagellate Margalefidinium fulvescens (=Cochlodinium fulvescens) isolated from the Jiaozhou Bay, China. Acta Oceanol. Sin. 2018, 37, 11-17. [CrossRef]

6. Tomas, C.R.; Smayda, T.J. Red tide blooms of Cochlodinium polykrikoides in a coastal cove. Harmful Algae 2008, 7, 308-317. [CrossRef]

7. Kudela, R.M.; Gobler, C.J. Harmful dinoflagellate blooms caused by Cochlodinium sp.: Global expansion and ecological strategies facilitating bloom formation. Harmful Algae 2012, 14, 71-86. [CrossRef]

8. Richlen, M.L.; Morton, S.L.; Jamali, E.A.; Rajan, A.; Anderson, D.M. The catastrophic 2008-2009 red tide in the Arabian Gulf region, with observations on the identification and phylogeny of the fish-killing dinoflagellate Cochlodinium polykrikoides. Harmful Algae 2010, 9, 163-172. [CrossRef]

9. Kim, H. Cochlodinium polykrikoides blooms in Korean coastal waters and their mitigation. Harmful Algae 1998, 227-228.

10. Reñé, A.; Garcés, E.; Camp, J. Phylogenetic relationships of Cochlodinium polykrikoides Margalef (Gymnodiniales, Dinophyceae) from the Mediterranean Sea and the implications of its global biogeography. Harmful Algae 2013, 25, 39-46. [CrossRef]

11. López-Cortés, D.J.; Núñez Vázquez, E.J.; Dorantes-Aranda, J.J.; Band-Schmidt, C.J.; Hernández-Sandoval, F.E.; BustillosGuzmán, J.J.; Leyva-Valencia, I.; Fernández-Herrera, L.J. The State of Knowledge of Harmful Algal Blooms of Margalefidinium polykrikoides (a.k.a. Cochlodinium polykrikoides) in Latin America. Front. Mar. Sci. 2019, 6. [CrossRef]

12. Curtiss, C.C.; Langlois, G.W.; Busse, L.B.; Mazzillo, F.; Silver, M.W. The emergence of Cochlodinium along the California Coast (USA). Harmful Algae 2008, 7, 337-346. [CrossRef]

13. Whyte, J.N.C.; Haigh, N.; Ginther, N.G.; Keddy, L.J. First record of blooms of Cochlodinium sp. (Gymnodiniales, Dinophyceae) causing mortality to aquacultured salmon on the west coast of Canada. Phycologia 2001, 40, 298-304. [CrossRef]

14. Kim, D.; Oda, T.; Muramatsu, T.; Kim, D.; Matsuyama, Y.; Honjo, T. Possible factors responsible for the toxicity of Cochlodinium polykrikoides, a red tide phytoplankton. Comp. Biochem. Physiol. Part C Toxicol. Pharmacol. 2002, 132, 415-423. [CrossRef]

15. Tang, Y.Z.; Gobler, C.J. Characterization of the toxicity of Cochlodinium polykrikoides isolates from Northeast US estuaries to finfish and shellfish. Harmful Algae 2009, 8, 454-462. [CrossRef] 
16. Wang, H.; Hu, Z.; Shang, L.; Leaw, C.P.; Lim, P.T.; Tang, Y.Z. Toxicity comparison among four strains of Margalefidinium polykrikoides from China, Malaysia, and USA (belonging to two ribotypes) and possible implications. J. Exp. Mar. Biol. Ecol. 2020, 524, 151293. [CrossRef]

17. Iwataki, M.; Kawami, H.; Mizushima, K.; Mikulski, C.M.; Doucette, G.J.; Relox, J.R.; Anton, A.; Fukuyo, Y.; Matsuoka, K. Phylogenetic relationships in the harmful dinoflagellate Cochlodinium polykrikoides (Gymnodiniales, Dinophyceae) inferred from LSU rDNA sequences. Harmful Algae 2008, 7, 271-277. [CrossRef]

18. Kim, C.-J.; Kim, H.-G.; Kim, C.-H.; Oh, H.-M. Life cycle of the ichthyotoxic dinoflagellate Cochlodinium polykrikoides in Korean coastal waters. Harmful Algae 2007, 6, 104-111. [CrossRef]

19. Elbrchter, M. Dinophyte reproduction: Progress and conflicts. J. Phycol. 2010, 39, 629-632. [CrossRef]

20. Tang, Y.Z.; Gobler, C.J. The toxic dinoflagellate Cochlodinium polykrikoides (Dinophyceae) produces resting cysts. Harmful Algae 2012, 20, 71-80. [CrossRef]

21. Kim, C.-H.; Cho, H.-J.; Shin, J.-B.; Moon, C.-H.; Matsuoka, K. Regeneration from hyaline cysts of Cochlodinium polykrikoides (Gymnodiniales, Dinophyceae), a red tide organism along the Korean coast. Phycologia 2019, 41, 667-669. [CrossRef]

22. Thangaraj, P.; Park, T.G.; Ki, J.-S. Molecular cloning reveals co-occurring species behind red tide blooms of the harmful dinoflagellate Cochlodinium polykrikoides. Biochem. Syst. Ecol. 2017, 70, 29-34. [CrossRef]

23. Gu, H.; Wu, Y.; Lü, S.; Lu, D.; Tang, Y.Z.; Qi, Y. Emerging harmful algal bloom species over the last four decades in China. Harmful Algae 2021, 102059. [CrossRef]

24. Hattenrath-Lehmann, T.K.; Jankowiak, J.; Koch, F.; Gobler, C.J. Prokaryotic and eukaryotic microbiomes associated with blooms of the ichthyotoxic dinoflagellate Cochlodinium (Margalefidinium) polykrikoides in New York, USA, estuaries. PLoS ONE 2019, 14, e0223067. [CrossRef] [PubMed]

25. Cui, Y.; Chun, S.J.; Baek, S.S.; Baek, S.H.; Kim, P.J.; Son, M.; Cho, K.H.; Ahn, C.Y.; Oh, H.M. Unique microbial module regulates the harmful algal bloom (Cochlodinium polykrikoides) and shifts the microbial community along the Southern Coast of Korea. Sci. Total Environ. 2020, 721, 137725. [CrossRef] [PubMed]

26. Ahn, Y.-H.; Shanmugam, P.; Ryu, J.-H.; Jeong, J.-C. Satellite detection of harmful algal bloom occurrences in Korean waters. Harmful Algae 2006, 5, 213-231. [CrossRef]

27. Guo, S.; Zhu, M.; Zhao, Z.; Liang, J.; Zhao, Y.; Du, J.; Sun, X. Spatial-temporal variation of phytoplankton community structure in Jiaozhou Bay, China. J. Oceanol. Limnol. 2019, 37, 1611-1624. [CrossRef]

28. Zhai, X.-Y.; Deng, Y.-Y.; Sun, Y.-Y.; Leaw, C.-P.; Lim, P.-T.; Zhao, Z.-X.; Hu, Z.-X.; Tang, Y.-Z. Morphological and ultrastructural comparison and phylogenetic analyses for the East Asian and American/Malaysian ribotypes of Margalefidinium polykrikoides. Oceanol. Limnol. Sin. 2019, 50, 1252-1262, (In Chinese with English Abstract). [CrossRef]

29. Lin, S.; Hu, Z.; Deng, Y.; Shang, L.; Gobler, C.J.; Tang, Y.Z. An assessment on the intrapopulational and intraindividual genetic diversity in LSU rDNA in the harmful algal blooms-forming dinoflagellate Margalefidinium (= Cochlodinium) fulvescens based on clonal cultures and bloom samples from Jiaozhou Bay, China. Harmful Algae 2020, 96, 101821. [CrossRef]

30. Liu, S.; Chen, N. Advances in biodiversity analysis of phytoplankton and harmful algal bloom species in the Jiaozhou Bay. Mar. Sci. 2021, 45, 170-188, (In Chinese with English Abstract). [CrossRef]

31. Liu, S.; Gibson, K.; Cui, Z.; Chen, Y.; Sun, X.; Chen, N. Metabarcoding analysis of harmful algal species in Jiaozhou Bay. Harmful Algae 2020, 92. [CrossRef]

32. Stoeck, T.; Bass, D.; Nebel, M.; Christen, R.; Jones, M.D.; Breiner, H.W.; Richards, T.A. Multiple marker parallel tag environmental DNA sequencing reveals a highly complex eukaryotic community in marine anoxic water. Mol. Ecol. 2010, 19 (Suppl. 1), $21-31$. [CrossRef] [PubMed]

33. Callahan, B.J.; McMurdie, P.J.; Rosen, M.J.; Han, A.W.; Johnson, A.J.; Holmes, S.P. DADA2: High-resolution sample inference from Illumina amplicon data. Nat. Methods 2016, 13, 581-583. [CrossRef]

34. Callahan, B.J.; McMurdie, P.J.; Holmes, S.P. Exact sequence variants should replace operational taxonomic units in marker-gene data analysis. ISME J. 2017, 11, 2639-2643. [CrossRef]

35. Guillou, L.; Bachar, D.; Audic, S.; Bass, D.; Berney, C.; Bittner, L.; Boutte, C.; Burgaud, G.; de Vargas, C.; Decelle, J.; et al. The Protist Ribosomal Reference database (PR2): A catalog of unicellular eukaryote small sub-unit rRNA sequences with curated taxonomy. Nucleic Acids Res. 2013, 41, D597-D604. [CrossRef]

36. Kumar, S.; Stecher, G.; Li, M.; Knyaz, C.; Tamura, K. MEGA X: Molecular Evolutionary Genetics Analysis across Computing Platforms. Mol. Biol. Evol. 2018, 35, 1547-1549. [CrossRef]

37. Leigh, J.W.; Bryant, D.; Nakagawa, S. popart: Full-feature software for haplotype network construction. Methods Ecol. Evol. 2015, 6, 1110-1116. [CrossRef]

38. De Luca, D.; Piredda, R.; Sarno, D.; Kooistra, W. Resolving cryptic species complexes in marine protists: Phylogenetic haplotype networks meet global DNA metabarcoding datasets. ISME J. 2021, 15, 1931-1942. [CrossRef] [PubMed]

39. Wei, T.; Simko, V. R package "corrplot": Visualization of a Correlation Matrix (Version 0.84) 2017. Available online: https: //github.com/taiyun/corrplot (accessed on 31 August 2021).

40. Wickham, H. Ggplot2: Elegant Graphics for Data Analysis; Springer: New York, NY, USA, 2009.

41. Roselli, L.; Vadrucci, M.R.; Belmonte, M.; Ciciriello, P.; Rubino, F.; Ungaro, N.; Caroppo, C. Two-Stages bloom of Margalefidinium cf. polykrikoides in a Mediterranean shallow bay (Ionian Sea, Italy). Mar. Pollut. Bull. 2020, 151, 110825. [CrossRef] [PubMed] 
42. Cortes-Altamirano, R.; Alonso-Rodriguez, R.; Salas-de-Leon, D.A. Historical observations of algal blooms in Mazatlan Bay, Sinaloa, Mexico (1979-2014). PLoS ONE 2019, 14, e0210631. [CrossRef] [PubMed]

43. Burki, F.; Sandin, M.M.; Jamy, M. Diversity and ecology of protists revealed by metabarcoding. Curr. Biol. 2021, 31, R1267-R1280. [CrossRef]

44. Sunagawa, S.; Coelho, L.P.; Chaffron, S.; Kultima, J.R.; Labadie, K.; Salazar, G.; Djahanschiri, B.; Zeller, G.; Mende, D.R.; Alberti, A.; et al. Ocean plankton. Structure and function of the global ocean microbiome. Science 2015, 348, 1261359. [CrossRef] [PubMed]

45. Kopf, A.; Bicak, M.; Kottmann, R.; Schnetzer, J.; Kostadinov, I.; Lehmann, K.; Fernandez-Guerra, A.; Jeanthon, C.; Rahav, E.; Ullrich, M.; et al. The ocean sampling day consortium. GigaScience 2015, 4, 27. [CrossRef] [PubMed]

46. Santoferrara, L.; Burki, F.; Filker, S.; Logares, R.; Dunthorn, M.; McManus, G.B. Perspectives from Ten Years of Protist Studies by High-Throughput Metabarcoding. J. Eukaryot. Microbiol. 2020, 67, 612-622. [CrossRef] [PubMed]

47. Giner, C.R.; Balagué, V.; Krabberød, A.K.; Ferrera, I.; Reñé, A.; Garcés, E.; Gasol, J.M.; Logares, R.; Massana, R. Quantifying long-term recurrence in planktonic microbial eukaryotes. Mol. Ecol. 2019, 28, 923-935. [CrossRef]

48. Godhe, A.; Asplund, M.E.; Harnstrom, K.; Saravanan, V.; Tyagi, A.; Karunasagar, I. Quantification of diatom and dinoflagellate biomasses in coastal marine seawater samples by real-time PCR. Appl. Environ. Microbiol. 2008, 74, 7174-7182. [CrossRef] [PubMed]

49. Ki, J.S.; Jang, G.Y.; Han, M.S. Integrated method for single-cell DNA extraction, PCR amplification, and sequencing of ribosomal DNA from harmful dinoflagellates Cochlodinium polykrikoides and Alexandrium catenella. Mar. Biotechnol. 2004, 6, 587-593. [CrossRef]

50. Kudela, R.M.; Ryan, J.P.; Blakely, M.; Lane, J.Q.; Peterson, T.D. Linking the physiology and ecology of Cochlodinium to better understand harmful algal bloom events: A comparative approach. Harmful Algae 2008, 7, 278-292. [CrossRef]

51. Yamatogi, T.; Sakaguchi, M.; Iwataki, M.; Matsuoka, K. Seasonal occurrence and growth characteristics of a harmful dinoflagellate Cochlodinium polykrikoides in Usuka Bay. Jpn. J. Phycol. 2005, 53, 229-235. 